Editorial

\section{Validation of nut roasting}

\section{Editorial}

Manufacturers that process raw nuts into nut butters such as peanut butter or almond butter have the responsibility to manufacture the nut butter using a Hazard Analysis Critical Control Point plan. While there are numerous Critical Control Points in the plan, one of the most important is the roasting step, either using hot air or oil roasting. Oil roasting provides a medium where heat transfer is more direct through coverage of individual nuts in a consistent and uniform manner. Roasting in hot air, however, is more complicated and is affected by factors such as the air flow pattern within the roaster, the depth of the nuts passing through the roaster, the speed of the belt through the roaster, the temperature of the heated air, the size and density of the nuts, and the initial temperature of the nuts entering the roaster. Manufacturers generally are required to validate that the roasting process provides a five-log reduction of salmonellae and this typically is accomplished by using nuts inoculated with a surrogate Enterococcus faecium, which has a heat resistance exceeding that of most salmonellae.

Validation procedures for dry roasting involve placing inoculated nuts into "socks" that are introduced into the raw end of the roaster and capturing the socks at the roaster output. Enumerating the surrogate before and after roasting allows for the calculation of the $\log$ reduction. Historically, introduction of the socks at the raw end has been executed after the roaster is fully loaded, that is, after the heating zones (typically two or three) have a full bed of nuts and has thus, have established an operational condition of heated air flow. The question remains, however, as to the leading edge and trailing edge of the nuts in the roasting process, that is, those nuts that are exposed to the heated air when the belt traveling through the roaster is not completely loaded. The heated air will travel differently in the heating zones when portions of the belt are not fully covered in nuts. Heating performance, and thus, the log reduction of salmonellae, will be different under these conditions.
Volume I Issue 3 - 2014

\author{
Robert A Seward \\ Department of Food safety and quality, Seward Global \\ Consulting, USA \\ Correspondence: Robert A Seward, Seward Global \\ Consulting, III 5th Street SE, Washington DC, \\ 20003, USA, Tel 402-66I-9096, Email skipdee@gmail.com \\ Received: June 30, 2014 | Published: July 0I, 2014
}

Experience has shown that socks should be placed in the leading and trailing edges of nuts to validate the lethality under these lessthan-optimal heating conditions. Adjustments to bed depth and belt speed during the time it takes to fully load the belt in the heating zones may be required to ensure adequate lethality is achieved. Once the belt is fully loaded, bed depths may be increased and belt speed may be increased. Without taking into account the leading and trailing edges of the roasting process, the lethality during these periods of time likely will be over-estimated.

The likelihood that raw nuts will be uniformly contaminated at concentrations near $100.000 /$ gram is highly unlikely. Thus, the safety of dry roasting processes that have been validated without consideration of the leading and trailing edge is likely adequate. However, validating the lethality during the time that the roaster is not fully loaded is recommended to fully understand the safety of the dry roasting process.

\section{Acknowledgments}

None.

\section{Conflicts of interest}

Authors declare that there is no conflict of interest. 